\begin{tabular}{|c|l|}
\hline Title & Observation of the soft mode in the Sm-Ca * phase \\
\hline Author(s) & Bourny, Valéry; Fajar, A ndika; Orihara, Hiroshi \\
\hline Citation & $\begin{array}{l}\text { Physical Review E, 62(5), R5903-R5906 } \\
\text { https://doi.org/10.1103/PhySRevE.62.R5903 }\end{array}$ \\
\hline Issue Date & 2000-11 \\
\hline Doc URL & http://hdl.handle.net/2115/50766 \\
\hline Rights & ○ 2000 A merican Physical Society \\
\hline Type & article \\
\hline File Information & Phys. Rev. E 62, R5903-R5906.pdf \\
\hline
\end{tabular}

Instructions for use 


\title{
Observation of the soft mode in the $\mathrm{Sm}-C_{\alpha}^{*}$ phase
}

\author{
V. Bourny, A. Fajar, and H. Orihara \\ Department of Applied Physics, School of Engineering, Nagoya University, Furo-cho, Nagoya 464-8603, Japan
}

(Received 11 April 2000)

\begin{abstract}
We have performed the electro-optical measurements in an antiferroelectric liquid crystal 4-(1-methylheptyloxycarbonyl)phenyl 4' -octylcarbonyloxybiphenyl-4-carboxylate (MHPOCBC) and observed the soft mode in the chiral Sm- $C_{\alpha}^{*}$ phase. This is a direct consequence that the $\mathrm{Sm}-C_{\alpha}^{*}$ phase is induced by the condensation of a tilting mode. A Landau-type theory was developed to analyze frequency dispersions in the second-order response and it was found that the Sm- $A-\mathrm{Sm}-C_{\alpha}^{*}$ phase transition is close to a tricritical point.
\end{abstract}

PACS number(s): 64.70.Md, 61.30.Gd

Recently, the structure of the $\mathrm{Sm}-C_{\alpha}^{*}$ phase has been clarified by means of resonant $\mathrm{x}$-ray scattering by Mach et al. [1]. The Sm- $C_{\alpha}^{*}$ phase was found to be an incommensurate, short-period ferroelectriclike structure. Therefore, the $\mathrm{Sm}-C_{\alpha}^{*}$ phase is considered to be formed by the soft mode condensation at a general point, $q_{c}$, of the smectic Brillouin zone. The existence of the soft mode is necessary for this scenario of the phase transition. However, the soft mode in the Sm- $A$ phase cannot be observed by means of usual measurements. In the $\mathrm{Sm}-C_{\alpha}^{*}$ phase, on the other hand, the soft mode is expected to be observable with suitable measurements because of the symmetry breaking. In fact, in the antiferroelectric phase $\left(\mathrm{Sm}-C_{A}^{*}\right)$ several modes such as amplitude and phase modes were observed by the photoncorrelation [2], electro-optic [3], and nonlinear dielectric spectroscopies [4] due to a doubling of a unit cell. In actual measurements, it is important whether the relaxation frequency is within the observable frequency region or not. Fortunately, unusually large pretransitional fluctuations have been observed near the phase transition point between the $\mathrm{Sm}-A$ and $\mathrm{Sm}-C_{\alpha}^{*}$ phases by the high-resolution heat capacity measurement [5] and by the high-resolution birefringence measurement [6], indicating that the critical slowing down definitely takes place and the relaxation frequency may be small enough to be observable near the transition point.

Quite recently, we have reported the Kerr effect due to the $\mathrm{Sm}-A-\mathrm{Sm}-C_{\alpha}^{*}$ phase transition in MHPOBC [7]. The same behavior was observed also in the $\mathrm{C} 8$ tolan [8]. Generally, in the Sm- $A$ phase, the application of an electric field brings about the spatially uniform tilt of molecules due to the electroclinic effect, resulting only in the rotation of indicatrix, i.e., the Pockels effect. In the previous paper, however, a simple theory under a dc field was presented to explain the Kerr effect [7], though the actual electro-optical measurement was made under ac electric fields. In MHPOBC we have found a characteristic frequency dispersion in the second-order response [7], but the analysis of it was not adequately made because we had no theoretically derived formula for it.

In this Rapid Communication, we report an observation of the soft mode in the $\mathrm{Sm}-C_{\alpha}^{*}$ phase. First, we develop a phenomenological theory and then obtain the temperature dependence of the relaxation frequency from experimental results by utilizing the theory.
In electro-optical measurements of a homogeneous cell between crossed polarizers, where the $z$ axis is set to be along the layer normal and the $x$ axis along the electric field direction, the change of transmitted light intensity, $\Delta I$, due to an electric field is expressed as [8,9]

$$
\begin{aligned}
\Delta I / I_{0}= & 2 \sin 4 \alpha_{0} \sin ^{2}\left(\beta n_{a 0}\right) \Delta \alpha+4 \cos 4 \alpha_{0} \sin ^{2}\left(\beta n_{a 0}\right) \Delta \alpha^{2} \\
& +\beta \sin ^{2} 2 \alpha_{0} \sin \left(2 \beta n_{a 0}\right) \Delta n_{a},
\end{aligned}
$$

where $\beta=\pi d / \lambda, I_{0}$ is the incident light intensity, $d$ the sample thickness, $\lambda$ the wavelength of light, $\alpha_{0}$ and $n_{a 0}$ are the angle of the optical axis with respect to one of the polarizer directions and the anisotropy of refractive index in the absence of the field, respectively, and $\Delta \alpha$ and $\Delta n_{a}$ are electrically induced parts. Since $\Delta \alpha$ and $\Delta n_{a}$ are proportional to the field and the square of it, respectively, the first one can be detected in the first-order electro-optic response and the two others in the second-order one. By utilizing the fact that each term in Eq. (1) has different $\alpha_{0}$ dependence, we can suppress the $\Delta \alpha^{2}$ term by setting $\alpha_{0}$ at $22.5^{\circ}$ to measure only the $\Delta n_{a}$ term in the second-order response. The terms $\Delta \alpha, \Delta n_{a}$, and $n_{a 0}$ are calculated from the dielectric tensor, the components of which are [9]

$$
\begin{gathered}
\varepsilon_{y y}=\varepsilon_{\perp}+\varepsilon_{a}\left\langle\xi_{x}^{2}\right\rangle, \quad \varepsilon_{y z}=\varepsilon_{a}\left\langle\xi_{x}\right\rangle, \\
\varepsilon_{z z}=\varepsilon_{\|}-\varepsilon_{a}\left(\left\langle\xi_{x}^{2}\right\rangle+\left\langle\xi_{y}^{2}\right\rangle\right),
\end{gathered}
$$

where $\varepsilon_{\|}, \varepsilon_{\perp}$, and $\varepsilon_{a}$ are, respectively, the dielectric constants parallel and perpendicular to the molecule and the dielectric anisotropy $\varepsilon_{\|}-\varepsilon_{\perp}$ for the visible light, $\xi_{x}$ and $\xi_{y}$ are the order parameters, and $\langle\cdots\rangle$ indicates the spatial average. The above averages are calculated on the basis of the Landau-Khalatnikov equation as follows.

Here, we consider only the spatially homogeneous mode (the ferroelectric mode) and the soft mode inducing the Sm$C_{\alpha}^{*}$ phase, which are enough for our purpose. We express the spatially dependent order parameters $\xi_{x}(j d)$ and $\xi_{y}(j d)$, where $j$ is the layer number and $d$ the layer spacing, by these modes: $\quad \xi_{x}(j d)=\xi_{f x}+\xi_{1} \cos q_{c} j d-\xi_{2} \sin q_{c} j d, \quad \xi_{y}(j d)=\xi_{f y}$ $+\xi_{1} \sin q_{c} j d+\xi_{2} \cos q_{c} j d$, where $q_{c}$ is the wave number related to the Sm- $A-\mathrm{Sm}-C_{\alpha}^{*}$ phase transition, and $\left(\xi_{f x}, \xi_{f y}\right)$ and $\left(\xi_{1}, \xi_{2}\right)$ are, respectively, the ferroelectric and soft 
modes. Under an electric field along the $x$ axis the free energy can be expanded in the $\mathrm{Sm}-A$ phase

$$
\begin{aligned}
f= & \frac{\alpha}{2}\left(\xi_{1}^{2}+\xi_{2}^{2}\right)+\frac{\beta}{4}\left(\xi_{1}^{2}+\xi_{2}^{2}\right)^{2}+\frac{\lambda}{2}\left(\xi_{1}^{2}+\xi_{2}^{2}\right) \xi_{f x}^{2} \\
& -\frac{\varepsilon_{a}^{\prime}}{4}\left(\xi_{1}^{2}+\xi_{2}^{2}\right) E_{x}^{2}-c \xi_{f x} E_{x}+\frac{a}{2} \xi_{f x}^{2},
\end{aligned}
$$

where we have dropped $\xi_{f y}$ because it is not induced by the electric field along the $x$ axis, and $\varepsilon_{a}^{\prime}$ is the dielectric anisotropy at low frequencies. The $\lambda$ term represents the nonlinear coupling between the ferroelectric and soft modes. The coefficient $\alpha$ is assumed to be linearly dependent on the temperature and becomes zero at the transition point. From the equilibrium conditions under no field in the $\mathrm{Sm}-C_{\alpha}^{*}$ phase we have one solution: $\xi_{1}=\xi_{s}=\sqrt{-\alpha / \beta}, \xi_{2}=0, \xi_{f x}=\xi_{f y}=0$. In the Sm- $C_{\alpha}^{*}$ phase $\xi_{1}$ and $\xi_{2}$ may be changed by the applied field through the nonlinear coupling and the dielectric anisotropy and so they are expressed as $\xi_{1}=\xi_{s}+\Delta \xi_{1}, \xi_{2}=\Delta \xi_{2}$, where the field-induced parts, $\Delta \xi_{1}$ and $\Delta \xi_{2}$, represent the amplitude mode (the soft mode) and the phase mode (the Goldstone mode), respectively. Thus we have the free energy in the $\mathrm{Sm}-C_{\alpha}^{*}$ phase,

$$
\begin{aligned}
f= & f_{0}+\frac{\alpha+3 \beta \xi_{s}^{2}}{2} \Delta \xi_{1}^{2}+\lambda \xi_{s} \Delta \xi_{1} \xi_{f x}^{2}-\frac{1}{2} \varepsilon_{a}^{\prime} \xi_{s} \Delta \xi_{1} E_{x}^{2}-c \xi_{f x} E_{x} \\
& +\frac{a+\lambda \xi_{s}^{2}}{2} \xi_{f x}^{2},
\end{aligned}
$$

where we have omitted the higher-order terms which contribute nothing to the second-order electro-optic response, and $f_{0}$ is the irrelevant part. Note that the above free energy density does not include $\Delta \xi_{2}$, indicating that the relaxation frequency of the Goldstone mode should be zero and it should not be excited by an applied field. However, the soft mode $\Delta \xi_{1}$ can be exited.

The Landau-Khalatnikov equation in our system is

$$
\gamma \frac{d \xi_{f x}}{d t}=-\frac{\partial f}{\partial \xi_{f x}}, \quad \gamma \frac{d \Delta \xi_{1}}{d t}=-\frac{\partial f}{\partial \Delta \xi_{1}},
$$

where we have assumed that the viscosity coefficient for $\Delta \xi_{1}$ should be the same with the one for the ferroelectric mode. By solving the above set of equations under an ac field, $E_{x}$ $=E_{0} \cos \omega t$, we get up to the second order with respect to $E_{0}$,

$$
\begin{gathered}
\xi_{f x}=\operatorname{Re}\left[\chi_{f}(\omega) \exp (i \omega t)\right] c E_{0}, \\
\Delta \xi_{1}=\chi_{s}(0)\left(\frac{\varepsilon_{a}^{\prime}}{4}-\frac{1}{2} \lambda c^{2}\left|\chi_{f}(\omega)\right|^{2}\right) \xi_{s} E_{0}^{2} \\
+\operatorname{Re}\left[\chi_{s}(2 \omega)\left(\frac{\varepsilon_{a}^{\prime}}{4}-\frac{1}{2} \lambda c^{2} \chi_{f}(\omega)^{2}\right) \exp (i 2 \omega t)\right] \xi_{s} E_{0}^{2},
\end{gathered}
$$

with

$$
\begin{gathered}
\chi_{f}(\omega)=\left(a+\lambda \xi_{s}^{2}+i \omega \gamma\right)^{-1}, \\
\chi_{s}(\omega)=\left(\alpha+3 \beta \xi_{s}^{2}+i \omega \gamma\right)^{-1},
\end{gathered}
$$

where $\chi_{f}(\omega)$ and $\chi_{s}(\omega)$ are, respectively, the susceptibilities of the ferroelectric mode and the soft mode. From Eq. (2) we finally get [9]

$$
\begin{aligned}
\Delta n_{a}= & -\frac{\varepsilon_{a}}{2}\left(\frac{2}{\sqrt{\varepsilon_{\|}}}+\frac{1}{\sqrt{\varepsilon_{\perp}}}\right) \xi_{s} \Delta \xi_{1} \\
& +\frac{3 \varepsilon_{a}}{4}\left(\frac{1}{\sqrt{\varepsilon_{\|}}}+\frac{1}{\sqrt{\varepsilon_{\perp}}}\right) \xi_{s}^{2} \xi_{f x}^{2}, \Delta \alpha=\epsilon_{a} \xi_{f x} .
\end{aligned}
$$

It should be noted that the Kerr effect is induced not only by the soft mode but also by the ferroelectric mode.

The studied liquid crystal was MHPOCBC and its phase sequence as follows [10]: $\mathrm{K}-\mathrm{Sm}-I_{A}^{*}-\mathrm{Sm}-C_{A}^{*}-\mathrm{Sm}-$ $C_{\alpha}^{*}-\mathrm{Sm}-A-I$.

In the previous experiment [7], we used MHPOBC, which also has the $\mathrm{Sm}-A-\mathrm{Sm}-C_{\alpha}^{*}$ phase transition. In comparison with MHPOBC, however, MHPOCBC has a merit that the soft mode inducing the Sm- $A-\mathrm{Sm}-C_{\alpha}^{*}$ phase transition and the ferroelectric soft mode can be easily separated in the frequency domain, i.e., they are relatively different in relaxation frequency, as will be shown later.

The cells used in the experiments were of usual sandwich type, consisting of two parallel glass substrates with inner surfaces coated with indium tin oxide (ITO). An alignment layer of polyimid was deposited on the ITO. In order to obtain good alignment, the samples were submitted for one hour to a low frequency ac electric field around the Sm$A-\mathrm{Sm}-C_{\alpha}^{*}$ phase transition point. The cell thickness was 25 $\mu \mathrm{m}$. The experimental setup for the electro-optical measurements was the same as in our previous paper [7]. The cell was put between crossed polarizers of a polarizing microscope, and the polarizer direction was set at $\alpha_{0}=22.5^{\circ}$ with respect to the optical axis, where only the birefringence change can be detected in the second-order response. The frequency dispersions were measured from $100 \mathrm{~Hz}$ to $1 \mathrm{MHz}$ at stabilized temperatures by using a hot stage. Hereafter we use a complex intensity $\Delta I$ defined as $\Delta I=a \exp (i \phi)$, where $a$ and $\phi$ are, respectively, the amplitude and the phase in each response.

The typical frequency dispersions of the first-order response $\Delta I_{1 f}$ and the second-order response $\Delta I_{2 f}$ in the Sm- $A$ phase are shown in Fig. 1. In the first response [Fig. 1(a)], only one Debye-type relaxation is involved in the measured frequency region, which is the ferroelectric mode, as is seen from Eqs. (8) and (9). The solid line represents the best fit by $\Delta I_{1 f}(\omega)=A_{f} /\left(1+i \omega \tau_{f}\right)$, where $\tau_{f}=\gamma /\left(a+\lambda \xi_{s}^{2}\right)$ is the relaxation time of the ferroelectric mode. In the second-order response [Fig. 1(b)], on the other hand, the squared Debyetype relaxation is observed. This relaxation originates in the ferroelectric mode, as is seen from Eq. (9), in which $\xi_{s}^{2}$ should be replaced by the thermal average of $\xi^{2}$ in the Sm- $A$ phase. The Kerr effect due to the thermal fluctuation has been observed also in MHPOBC [7]. The solid line in Fig. 1(b) is the best fit by the squared Debye. It was confirmed that the relaxation frequencies obtained from the first- and second-order responses are the same. 


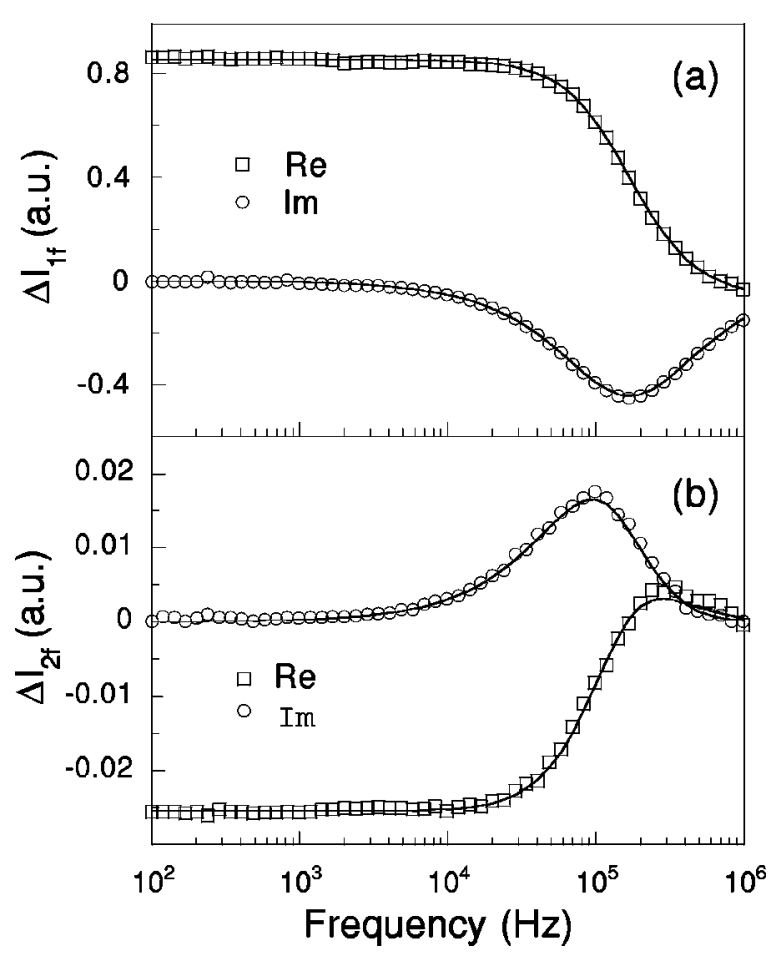

FIG. 1. Typical frequency dispersions of (a) the first-order response $\Delta I_{1 f}$ and (b) the second-order response $\Delta I_{2 f}$ obtained at $\alpha_{0}=22.5^{\circ}$ in the Sm- $A$ phase $\left(T=104.1^{\circ} \mathrm{C}\right)$.

In Fig. 2 the typical frequency dispersions in the $\mathrm{Sm}-C_{\alpha}^{*}$ phase are shown. In the first-order response [Fig. 2(a)], only the ferroelectric mode is observed as well as in the $\mathrm{Sm}-A$ phase. In the second-order response [Fig. 2(b)], on the con-

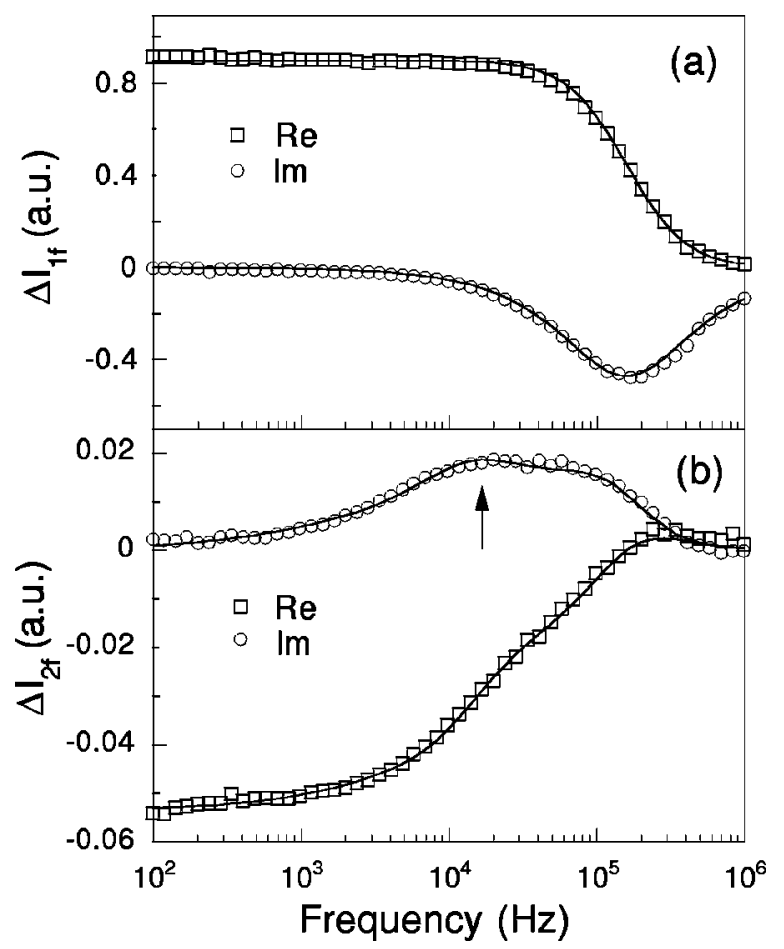

FIG. 2. Typical frequency dispersions of (a) the first-order response $\Delta I_{1 f}$ and (b) the second-order response $\Delta I_{2 f}$ obtained at $\alpha_{0}=22.5^{\circ}$ in the $\mathrm{Sm}-C_{\alpha}^{*}$ phase $\left(T=103.75^{\circ} \mathrm{C}\right)$. The soft mode is indicated by an arrow.

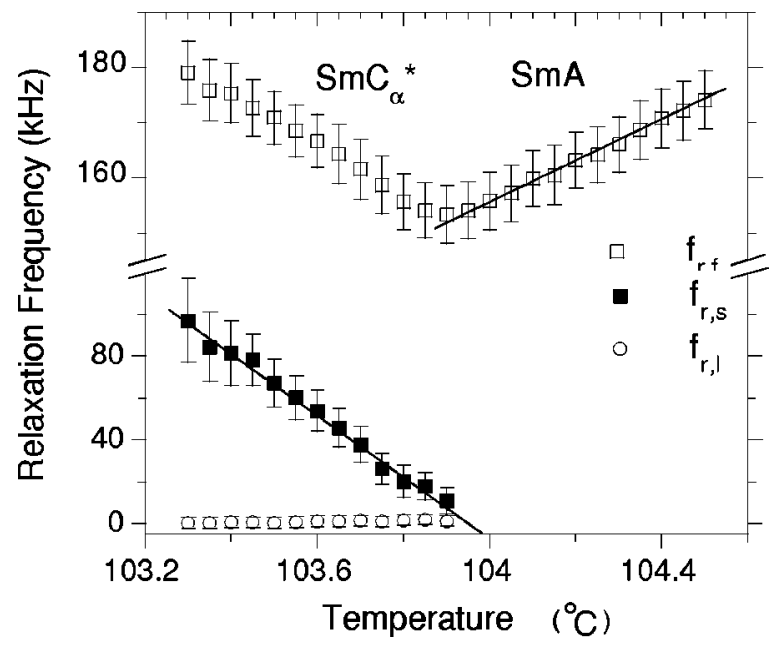

FIG. 3. Temperature dependencies of the relaxation modes obtained from electro-optical measurements. $f_{r, f}$ and $f_{r, s}$ are the relaxation frequencies of the ferroelectric mode and the soft mode respectively, and $f_{r, l}$ is that of a low-frequency mode.

trary, another mode is seen [indicated by an arrow in Fig. 2(b)]. This mode should be the soft mode related to the Sm$A-\mathrm{Sm}-C_{\alpha}^{*}$ phase transition. From Eqs. (7)-(9), it is easily seen that the dispersion in the $\mathrm{Sm}-C_{\alpha}^{*}$ can be analyzed by the formula:

$$
\begin{aligned}
\Delta I_{2 f}(\omega)= & \frac{1}{1+i 2 \omega \tau_{s}} \cdot\left(A_{s 1}+\frac{A_{s 2}}{\left(1+i \omega \tau_{f}\right)^{2}}\right)+\frac{A_{f 2}}{\left(1+i \omega \tau_{f}\right)^{2}} \\
& +\frac{A_{l}}{1+\left(i \omega \tau_{l}\right)^{\beta_{l}}}
\end{aligned}
$$

where $\tau_{s}=\gamma /\left(\alpha+3 \beta \xi_{s}^{2}\right)$ is the relaxation time of the soft mode, and note that the last term has been added so as to fit to a low-frequency mode appearing in the experiment, though the origin of it is not yet clear. We had a good agreement between the experiment and the theory [the solid line in Fig. 2(b)], where in the fitting we fixed $\tau_{f}$ to be the same value determined from the first-order response.

Figure 3 shows the temperature dependencies of the relaxation frequencies, $f_{r, s}=\left(2 \pi \tau_{s}\right)^{-1}, f_{r, f}=\left(2 \pi \tau_{f}\right)^{-1}$, and $f_{r, l}=\left(2 \pi \tau_{l}\right)^{-1}$. In the Sm- $C_{\alpha}^{*}$ phase the soft mode, $f_{r, s}$, related to the transition is clearly seen, and the partial softening of the ferroelectric mode, $f_{r, f}$, is also seen. The Curie Weiss low is observed for the soft mode and the ferroelectric mode. The extrapolation of the soft mode frequency gives $T_{C}=103.95^{\circ} \mathrm{C}$. Here, it should be noted that the slope of the soft mode $\left(145 \mathrm{kHz} /{ }^{\circ} \mathrm{C}\right)$ in the $\mathrm{Sm}-C_{\alpha}^{*}$ phase is about four times as large as the one of the ferroelectric mode $(36 \mathrm{kHz} /$ ${ }^{\circ} \mathrm{C}$ ) in the $\mathrm{Sm}-A$ phase. Assuming that in the $\mathrm{Sm}-A$ phase the slope of the soft mode, which is not experimentally obtained now, should be the same with that of the ferroelectric mode, the above result indicates that the $\mathrm{Sm}-A-\mathrm{Sm}-C_{\alpha}^{*}$ phase transition should be close to the tricritical point, where the fourth-order coefficient of the Landau expansion vanishes [11], as has been also pointed out by Skarabot et al. [6]. This assumption may be justified by the discrete model proposed by Sun et al. [12]. In the model the transitions to 
tilted phases from the Sm- $A$ phase take place in each layer even without interactions between neighboring layers, leading to the result that the doubly degenerate dispersion branch in the Sm- $A$ phase goes down without changing the shape as the temperature is decreased, i.e., the slope of the relaxation frequency versus the temperature measured at any point in the smectic Brillouin zone should be the same. From the above result it is necessary to include the sixth-order term. Here, we have the sign of the coupling constant $\lambda$, comparing the slopes of the ferroelectric mode in the Sm- $A$ and Sm$C_{\alpha}^{*}$ phases. From Eq. (8) it is seen that the relaxation frequency of the ferroelectric mode is proportional to $a+\lambda \xi_{s}^{2}$. The experimental result that the slope in the $\mathrm{Sm}-C_{\alpha}^{*}$ is larger than the one in the Sm- $A$ phase leads to the positive $\lambda$. This is directly obtained from the fitting results, in which $A_{s 2}$ (the same sign with $\lambda)>0$. In addition, we get $\varepsilon_{a}^{\prime}$ (the same sign with $\left.A_{s 1}\right)<0$.

In conclusion, we have observed the soft mode in the Sm$C_{\alpha}^{*}$ phase, which is a direct consequence of the soft mode condensation. The temperature dependencies of the relaxation frequencies indicate that the Sm- $A-\mathrm{Sm}-C_{\alpha}^{*}$ phase transition should be close to the tricritical point. The full analyses taking into account the sixth-order terms in the free energy of the discrete model will be presented in the future.

We would like to thank Showa Shell Sekiyu Co. Ltd. for supplying MHPOCBC. One of the authors (V.B.) thanks the Japan Society of Promotion of Science. This study was partly supported by a Grant-in Aid from the Ministry of Education, Science, Sports and Culture (Grant No. 11099724).
[1] P. Mach, R. Pindak, A.-M. Levelut, P. Barois, H. T. Nguyen, C. C. Huang, and L. Furenlid, Phys. Rev. Lett. 81, 1015 (1998).

[2] I. Muševič, R. Blinc, B. Žekš, M. Čopič, M. M. Wittebrood, Th. Rasing, H. Orihara, and Y. Ishibashi, Phys. Rev. Lett. 71, 1180 (1993).

[3] K. Hiraoka, H. Takezoe, and A. Fukuda, Ferroelectrics 147, 13 (1993).

[4] K. Obayashi, H. Orihara, and Y. Ishibashi, J. Phys. Soc. Jpn. 64, L3188 (1995).

[5] K. Ema and H. Yao, Phys. Rev. E 57, 6677 (1998).

[6] M. Škarabot, K. Kocevar, R. Blinc, G. Heppke, and I. Musevic, Phys. Rev. E 59, R1323 (1999).
[7] A. Fajar, H. Orihara, V. Bourny, J. Pavel, and V. Lorman, Jpn. J. Appl. Phys. 39, L166 (2000).

[8] V. Bourny, J. Pavel, V. Lorman, and H. T. Nguyen, Liq. Cryst. 27, 559 (2000).

[9] H. Orihara and Y. Ishibashi, J. Phys. Soc. Jpn. 64, 3775 (1995).

[10] T. Isozaki, Y. Suzuki, I. Kawamura, K. Mori, N. Nakamura, N. Yamamoto, Y. Yamada, H. Orihara, and Y. Ishibashi, Jpn. J. Appl. Phys. 30, L1573 (1991).

[11] R. Blinc and B. Zeks, Soft Modes in Ferroelectrics and Antiferroelectrics (North-Holland, Amsterdam, 1974).

[12] H. Sun, H. Orihara, and Y. Ishibashi, J. Phys. Soc. Jpn. 62, 2706 (1993). 